\title{
Posterior interosseous neuropathy in polo riders a specific sport-related mononeuropathy
}

\section{Abstract}

Posterior interosseous neuropathy is an entrapment of the deep branch of the radial nerve in the forearm. The deep branch of the radial nerve is primarily a motor nerve, and compression of this nerve may lead to weakness in finger and thumb extension without sensory abnormalities. We present two cases of polo riders suffering of posterior interosseous neuropathy, confirmed both by electrodiagnostic testing and by surgical exploration in one case. We review the anatomic course and distribution of the posterior interosseous nerve, the clinical picture of its injury, and discuss the probable mechanism of entrapment in polo players.
Volume 8 Issue 6 - 2017

\author{
Bendersky Mariana, ${ }^{1,2,4}$ Cagnone Juan \\ Carlos, ${ }^{3}$ Rey Roberto Danie,,2 Gómez \\ César ${ }^{1}$ \\ 'Department of Normal Anatomy, Living Anatomy Laboratory, \\ University of Buenos Aires, Argentina \\ ${ }^{2}$ Argentine Institute of Neurological Investigations, Argentina \\ ${ }^{3}$ Department of Upper Limb, Finocchietto Clinic, Argentina \\ ${ }^{4}$ Buenos Aires Italian Hospital, Intraoperative Neurophysiology \\ Unit, Argentina
}

Correspondence: Dra. Mariana Bendersky, Living Anatomy Laboratory, III Normal Anatomy Department, University of Buenos Aires, School of Medicine. Paraguay 2155, Ciudad Autonoma de Buenos Aires, Argentina, Tel 54 (I I) 5950-9605 Email mbendersky@fmeb.uba.ar

Received: June 16, 2017 | Published: September 08, 2017

\section{Introduction}

Posterior interosseous (PIoN) neuropathy is an entrapment of the deep branch of the radial nerve in the arcade of Frohse, a fibrous arch shaped by the supinator muscle. It accounts for less than $0.7 \%$ of all upper limb nerve compression syndromes..$^{1-7}$ The clinical picture is characterized by weakness of the posterior forearm muscles, with normal sensory examination. ${ }^{2,5-7}$

Elbow or forearm lesions reported so far in polo players were usually due to direct trauma. ${ }^{8}$ The most common movement in polo riders is alternative pronosupination with the right arm, and forced pronation or supination with the riding arm. Such muscular overuse may lead to injury to PIoN.

\section{Case reports}

\section{Case report $\mathbf{n}^{\circ} \mathrm{I}$}

NR, a 38-year-old healthy male, presented with progressive finger extension weakness. He had been training during the amateur polo session, when he noticed pain at the lateral side of his left elbow. The next few days he could not extend completely his third finger. Three months after onset, he reported pain at the left elbow and weakness of wrist and finger extensors. On physical examination, he showed subtle muscle atrophy in the left posterior forearm. Strength examination was $2 / 5$ in extension of the metacarpophalangeal joints. Wrist extension was $3 / 5$, with radial deviation (Figure 1). Sensation and reflexes were intact. Electrodiagnostic studies revealed an axonaldemyelinating lesion of the left PIoN at the elbow, with sparing of proximal radial innervated muscles and preservation of sensory radial nerve. Magnetic resonance imaging (MRI) of the left elbow showed abnormal signal intensity in extensorum digitorum communis and indicis proprius, suggestive of denervation. The patient underwent descompressive surgery, and intraoperative findings confirmed the entrapment (Figure 2). At 6 months follow-up, he was almost completely recovered.

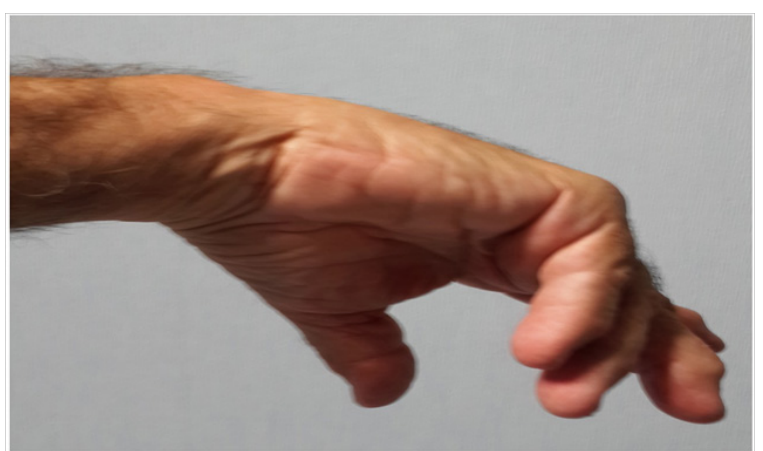

Figure I Clinical findings in patient $\mathrm{n}^{\circ} 2$, with weakness of wrist and fingers extensors. Middle and terminal phalanges are spared.

\section{Case report $n^{\circ} 2$}

HE, a 54 years-old healthy male, noticed a progressive loss of strength in fingers extension and thumb abduction during the last year, beginning in the last three fingers and later involving the others. He was an amateur polo player, and he also used to play tennis. On presentation, he had tenderness over the lateral epycondile, 2/5 weakness of wrist extensors and the metacarpophalangeal joints 2 nd to 5 th, and radial deviation of the hand (Figure 1). Electrodiagnostic tests and MRI showed similar results to those of patient $n^{\circ} 1$. The patient was then referred for surgical decompression, with full recovery at 1 year follow-up.

\section{Discussion}

The clinical picture of PIoN neuropathy can be explained by its anatomical features. ${ }^{1-6}$ PIoN first innervates and then pierces the supinator muscle. At the distal end of the arcade of Frohse, it splits into a medial branch, for Extensorum carpi ulnaris, digitorum communis and digiti minimi and a lateral branch, for Extensor pollicis longus and brevis, Abductor pollicis longus and Extensor indicis proprius. ${ }^{2,3}$ PIoN 
neuropathy weakens the extension and abduction of the thumb and the extension of the proximal phalanges. Middle and terminal phalanges are spared (Figure 1) because they are extended by the Interossei and Lumbricalis, innervated by the ulnar nerve. The radial deviation of the wrist is due to predominance of Extensor carpi radialis brevis, whose motor branch arises above the radial tunnel. Supination is not affected because it can be made with the elbow in some degree of flexion, by the biceps. Muscular paralysis can be simultaneous for all muscles, or in a sequential manner.

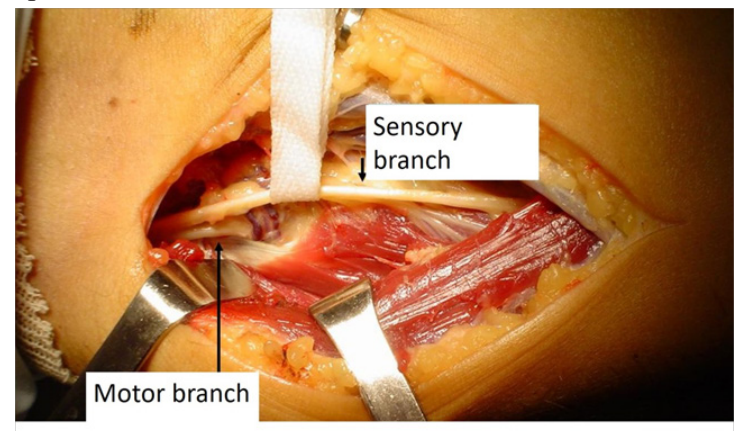

Figure 2 Surgical release of posterior interosseous nerve in patient $\mathrm{n}^{\circ} \mathrm{I}$. The nerve was degenerated, with atrophy and inflammation, and entrapped by a fibrous edge of the arcade of Frohse.

Differential diagnoses include: Higher paralysis of radial nerve (it affects brachiorradialis, and there is hypoesthesia in the radial territory of the hand). Saturnism (Usually accompanied by hematological disorders, spots on the gums, and a positive history of plumb exposition).

Disruption of the digital extensor tendons (wrist flexion can't elicit passive phalangeal extension. In radial palsies, undamaged tendons can passively extend the metacarpo-phalangic joints with this maneuver).

Electrodiagnostic studies helps localizing the nerve injury to the PioN, excluding other disorders, determining the severity of injury and predicting prognosis. ${ }^{5,7}$ Pain and tenderness at the lateral aspect of the elbow resemble the symptoms of lateral epycondilitis, due to compression of the recurrent epicondylar branch, and may be aggravated by forced supination. This syndrome results from repeated indirect trauma by forceful supination as the predisposing factor, and it has been reported in other sports or occupations requiring repetitive actions of the forearm muscles. ${ }^{4-6}$ Trauma to the nerve is possibly caused by repetitive alternation of pronation and supination, when the PIoN is displaced. ${ }^{5}$ In supination the PIoN moves laterally away from the midline and lenghtens. If the supinator muscle is tight, it can passively compress the nerve in pronation. ${ }^{1-3}$ A study by Werner. ${ }^{9}$ showed pressures of up to $200 \mathrm{mmHg}$ compressing the PIoN during active contraction of the supinator. The probable injury mechanism in patient $n^{\circ} 1$ could have been his habit of gripping the reins too hard, in forced supination with his left arm (Figure 3). Patient $n^{\circ} 2$, who played both tennis and polo, developed PIoN neuropathy in the swinging arm, probably due to a poor technique or over training.

Conservative treatment may be useful in mild and acute cases, when no identifiable cause is seen on imaging studies. If there is no improvement in 6 months, surgery is recommended. Prognosis usually depends on the timing of decompression: chronic lesions, with axonal impairment in nerve conduction studies, have less chances of recovery. ${ }^{10}$ Motor recovery follows the "rule of 18 ": motor recovery won't happen past 18 inches from the nerve injury after 18 months have passed, because nerves regenerate at about one inch per month, and motor endplates degenerate 18 months after nerve damage. ${ }^{2,5,7,10}$
Proximal branches of the nerve recover sooner than distal ones, so the pattern of strength recovery is inverse to that of the setting up of the weakness.

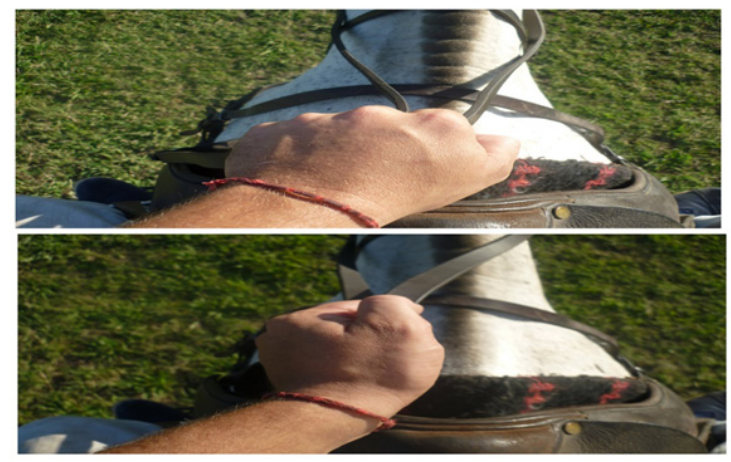

Figure 3 Gripping the reins too hard, in forced supination or pronation, as showed in these pictures, can compress the posterior interosseous nerve against the fibrous edge of the supinator muscle.

Stabilizing oneself on a $500 \mathrm{~kg}$ horse during sudden stops-andstarts from galloping at $65 \mathrm{~km} / \mathrm{h}$, sudden changes in direction, and twisting and leaning down to swing the mallet places a great deal of stress on many muscles, ligaments, and tendons. ${ }^{8}$ Applying the right technique for riding and swinging seems to be a good prevention for PIoN neuropathy, among other polo lesions.

\section{Acknowledgments}

None.

\section{Conflicts of interest}

None.

\section{References}

1. Riffaud L, Morandi X, Godey B et al. Anatomic bases for the compression and neurolysis of the deep branch of the radial nerve in the radial tunnel. Surg Radiol Anat. 1999;21(4):229-233.

2. Spinner $M$ The arcade of Frohse and its relationship to posterior interosseous nerve paralysis. J Bone Joint Surg. 1968;50(6):809-812.

3. Portilla Molina AE, Bour C, Oberlin C et al. The posterior interosseous nerve and the radial tunnel syndrome: an anatomical study. International Orthopaedics. 1998;22(2):102-106.

4. Dang AC, Rodner CM Unusual Compression Neuropathies of the Forearm, Part I: Radial Nerve. Journal of Hand Surgery. 2009;34(10):1906-1914.

5. Carfi J, Ma DM Posterior interosseous syndrome revisited. Muscle \& Nerve. 1985;8(6):499-502.

6. Cravens G, Kline DG Posterior Interosseous Nerve Palsies. Neurosurgery. 1990;27(3):397-402.

7. Fardin P, Negrin P, Spartá S Posterior interosseous nerve neuropathy. Clinical and electromyographical aspects. Electromyogr Clin Neurophysiol. 1992;32(4-5):229-234.

8. Costa Paz M, Aponte Tinao L, Muscolo DL Injuries to polo riders; a prospective evaluation. Brit J Sports Med. 1999;33(5):329-332.

9. Werner CO, Haeffner F, Rosen I Direct recording of local pressure in the radial tunnel during passive stretch and active contraction of the supinator muscle. Archives of Orthopaedic and Traumatic Surgery. 1980;96(4):299-301.

10. Tyser AR, Means KR Nerve Injuries About the Elbow: Treatment Options. Current Orthopaedic Practice. 2012;23(1):29-33. 